\title{
Chest Roentgenogram Classification and Clinical Parameters in Patients with Active Pulmonary Tuberculosis
}

\author{
Takanao Murate $^{1,2)}$, Kaoru Shimokata ${ }^{1)}$, Atsushi Watanabe ${ }^{1)}$, Satoshi Ichiyama $^{1)}$, \\ Hiroshi SaIto $^{1,3)}$, Sadaaki Yamori ${ }^{1,3)}$, Fumio Nomura ${ }^{1,3,4)}$, Tsuyoshi Iwahara ${ }^{4)}$, \\ Shuzo SAKaI ${ }^{4)}$, Kazuo NAKANISHI ${ }^{5)}$, Keisuke NishiwaKI ${ }^{5)}$ and Tatsuo OHGIMI ${ }^{6)}$
}

The relationship between the clinical parameters and chest film appearance was studied in patients with active pulmonary tuberculosis. Patients with extended disease were often malnourished and had weak tuberculin reaction accompanied by lymphopenia. They excreted a large amount of mycobacterial bacilli and a longer period was required for negative conversion of sputum culture. We confirmed that the criteria for chest roentgenogram classification established by the Japanese Society for Tuberculosis ("Gakkai Classification") is useful in the estimation of the risk of infection and the prognosis of tuberculosis patients.

(Internal Medicine 31: 185-188, 1992)

Key words: Gakkai Classification, malnutrition, tuberculin reaction, lymphopenia, infectiousness, prognosis

\section{Introduction}

In Japan, the "Gakkai Classification" has been widely used to evaluate patients with pulmonary tuberculosis. This classification designed to evaluate disease severity and infectiousness (1). Here, we clarified the relationship between chest film appearance as classified by "Gakkai Classification" and the clinical parameters in tuberculosis patients.

\section{Methods}

\section{Gakkai Classification}

The Japanese Society for Tuberculosis established the "Gakkai Classification" in 1959 (1). According to this classification, tuberculous lesions are classified as follows by chest roentgenogram findings: Extent 1, morbid foci that do not exceed the area from the lung apex to the horizontal line on front upper edge of second rib; Extent 3, morbid foci that exceed the hemi-thorax area; Extent 2, morbid foci between Extent 1 and Extent 3; Type I, widespread cavities which exceed the area of Extent 1 and morbid foci which exceed hemi-thorax area in total; Type II, morbid foci with cavities other than Type I; Type III, active infiltrative morbid foci with no cavities.

\section{Patients}

We studied 104 tubercle bacilli-positive patients (76 males, 28 females; $17-89$ year old) with pulmonary tuberculosis prospectively from July 1988 to April 1989. According to the "Gakkai Classification," they were classified; Type I, 7; Type II, 63; Type III, 34; Extent 1, 18; Extent 2, 61; Extent 3, 25. All patients were treated with isoniazid and rifampicin. Moreover, smearpositive case were additionally treated with ethambutol or streptomycin.

Clinical parameters

For the clinical parameters, age, sex, height, weight, Broca index, erythrocyte sedimentation rate (ESR), serum protein and albumin concentration, circulating white blood cell (WBC) and lymphocyte counts, tuberculin reaction, Gaffky scale of sputum, negative conversion term (the time from start of anti-tuberculosis therapy until achievement of negative conversion of sputum culture), complications and past histories were adopted.

\footnotetext{
From ${ }^{1)}$ First Department of Internal Medicine, Nagoya University School of Medicine, Nagoya, ${ }^{2)}$ Meitetsu Hospital, Nagoya, ${ }^{3)}$ National Chubu Hospital, Obu, ${ }^{4}$ Japanese Red Cross Nagoya First Hospital, Nagoya, ${ }^{5)}$ Social Insurance Chukyo Hospital, Nagoya, ${ }^{6}$ Aichi Prefectural Aichi Hospital, Okazaki

Received for publication September 11, 1990; Accepted for publication August 26, 1991

Reprint requests should be addressed to Kaoru Shimokata, MD, First Department of Internal Medicine, Nagoya University of School of Medicine, 65 Tsurumai-cho, Showa-ku, Nagoya 466, Japan.
} 


\section{Murate et al}

\section{Statistical analysis}

The analysis of variance and the chi-square test were usd.

\section{Results}

Age and Sex

Patients with no cavities were older than those with cavities (Fig. 1). Extent did not relate to age. Type and extent did not relate to sex (data not shown).

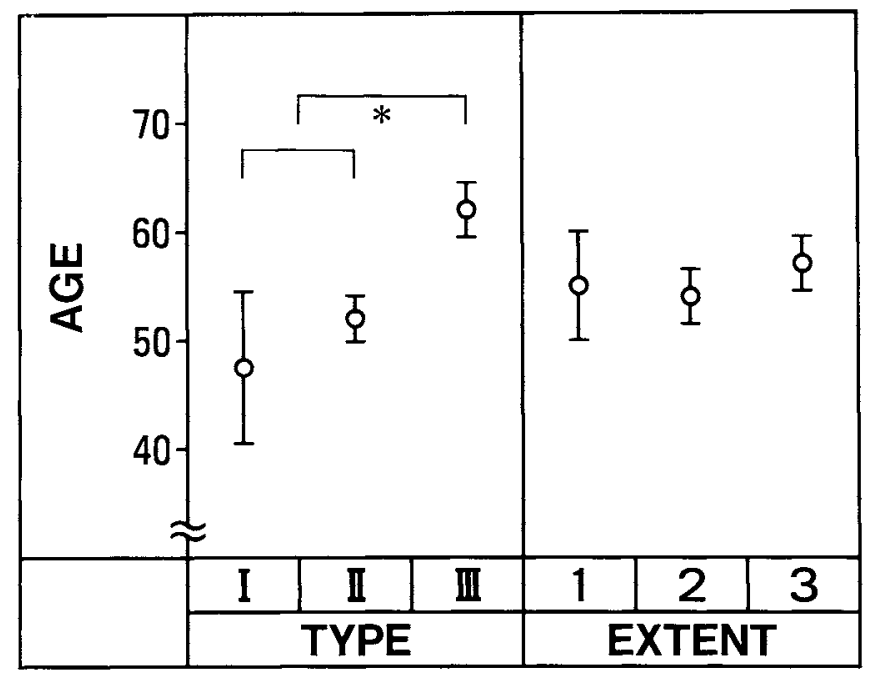

Fig. 1. Relationship between type, extent and age. circle, average; bar, standard error. ${ }^{*} \mathrm{p}<0.05$.

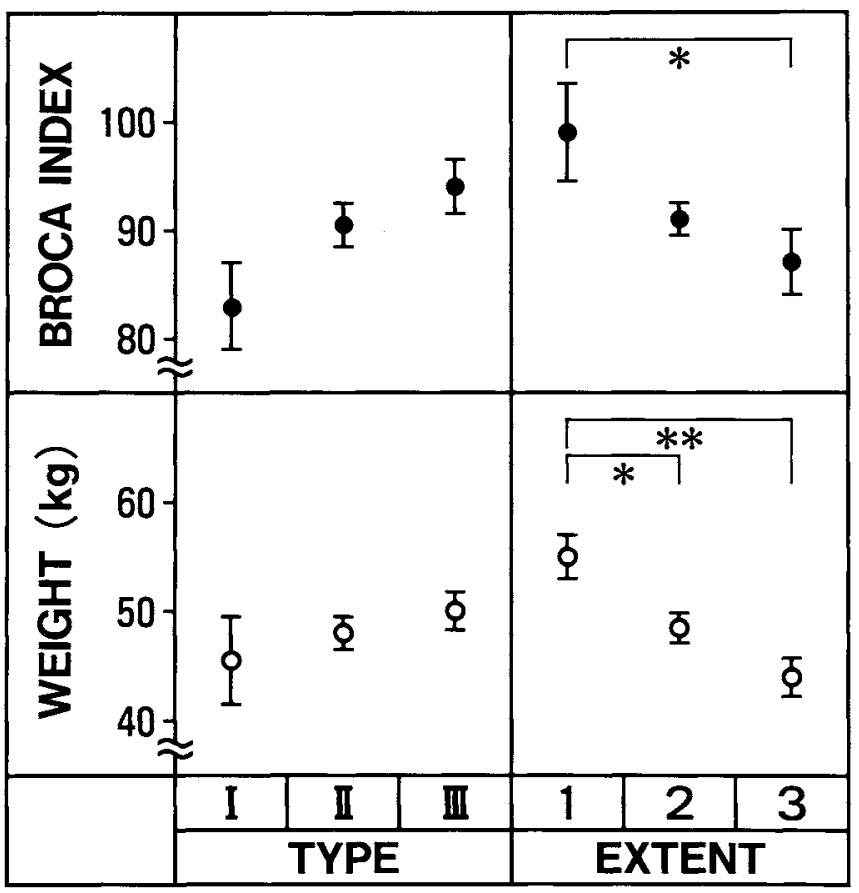

Fig. 2. Relationship between type, extent, weight and Broca index. circle, average; bar, standard error. ${ }^{*} \mathrm{p}<0.05,{ }^{* *} \mathrm{p}<0.01$.

\section{Nutritional parameters}

Weight and Broca index were small in the extended cases (Fig. 2). Serum albumin concentration was decreased in the patients with a more severe type or extent (Fig. 3).

\section{Immunological parameters}

There were no differences in WBC count. However, severe lymphopenia was observed in Extent 3 (Fig. 4). Tuberculin skin reaction (maximal diameter of erythema) was reduced in Type I or Extent 3 (Fig. 5). Doublecontour erythema by tuberculin skin test was observed in 7 of Extent 1, 16 of Extent 2 and one of Extent 3; it was reduced in the extended cases $(p<0.05)$. The frequency of vesicle formation in the tuberculin skin test did not differ (data not shown).

\section{ESR, Gaffky scale and negative conversion term}

ESR was raised in Extent 2 and Extent 3 patients (Fig. 6). The Gaffky scale was low in patients with no cavities and high in the extended cases. The negative conversion term differed similarly (Fig. 7).

\section{Complications and past histories}

There were no differences regarding the past histories including gastrectomy, malignancy, diabetes mellitus, liver dysfunction, rheumatoid disease and pneumoconiosis. However, the complication of respiratory failure was frequent in Extent 3 cases $(p<0.01)$ (data not shown).

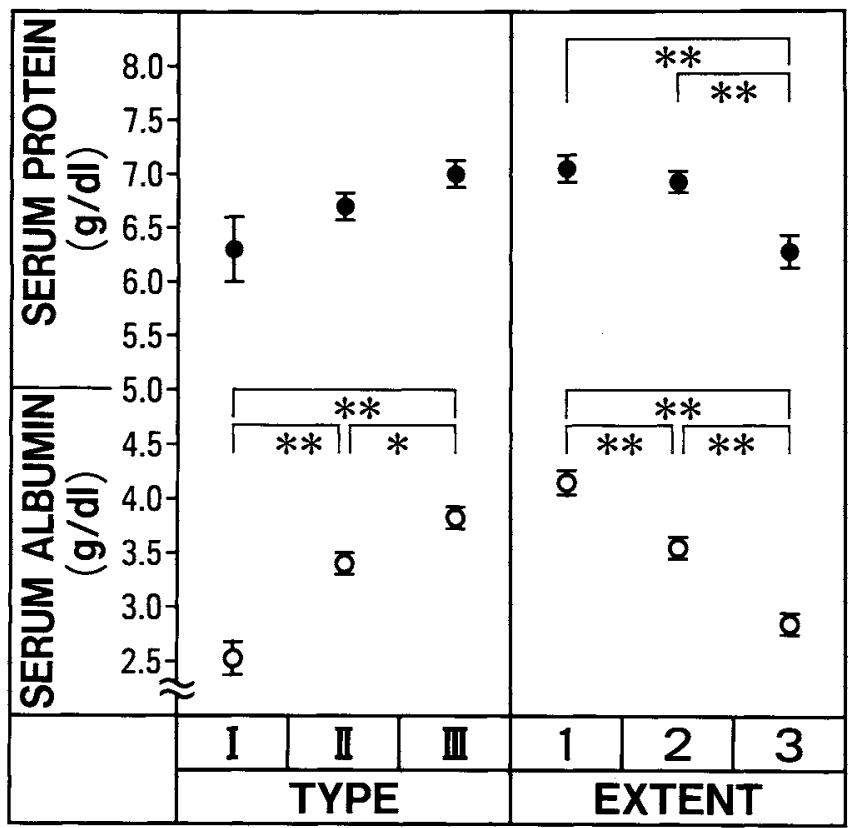

Fig. 3. Relationship between type, extent, serum protein and albumin concentration. circle, average; bar, standard error. ${ }^{*} p<0.05$, ${ }^{* * *} \mathrm{p}<0.01$. 


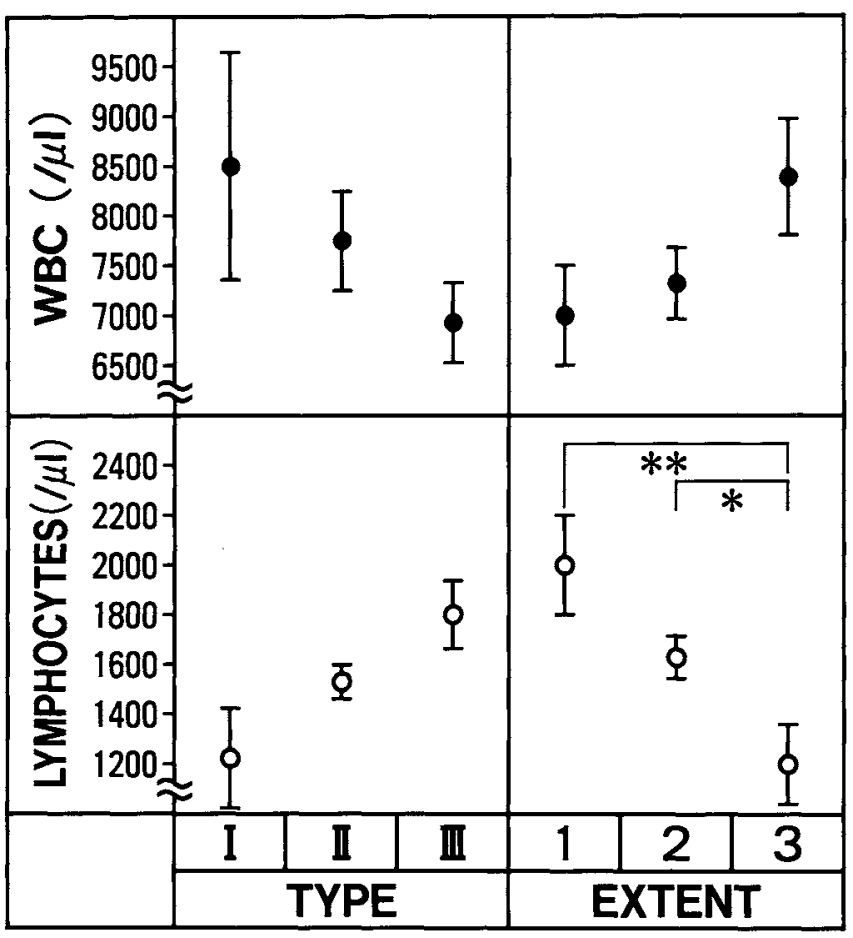

Fig. 4. Relationship between type, extent, circulating white blood cell and lymphocyte counts. circle, average; bar, standard error. ${ }^{*} \mathrm{p}<0.05,{ }^{* *} \mathrm{p}<0.01$

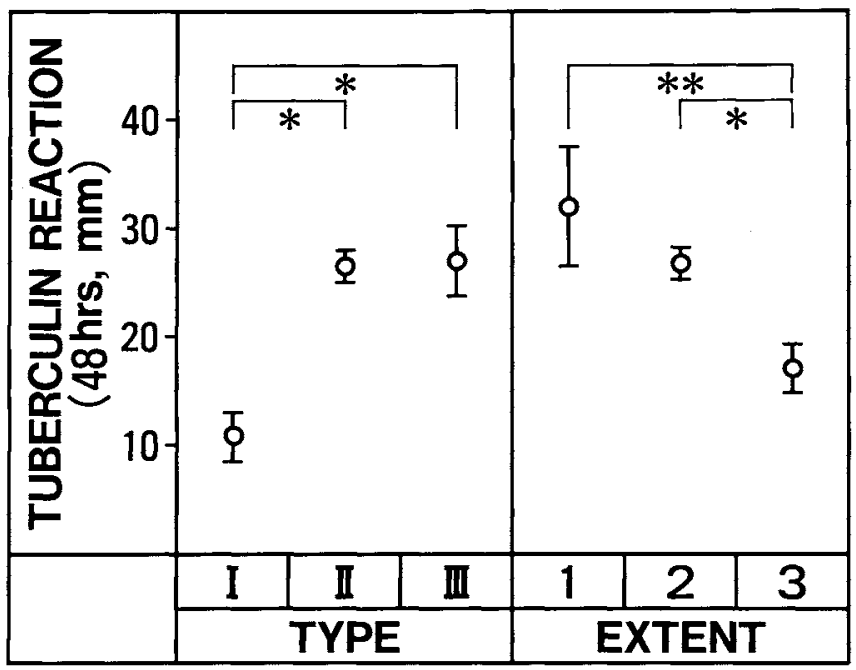

Fig. 5. Relationship between type, extent and tuberculin reaction (maximal diameter of erythema). circle, average; bar, standard error ${ }^{*} \mathrm{p}<0.05,{ }^{* *} \mathrm{p}<0.01$.

\section{Discussion}

In patients with tuberculosis, multiple defects in cellular immunity such as chemotaxis, phagocytosis and bactericidal capacities have been reported (2). Aging and malnutrition have been discussed as major clinical factors facilitating development of active tuberculosis.

Aged patients with tuberculosis have markedly reduced cellular immunity (3-5). Although the $\mathrm{T}$ cell population

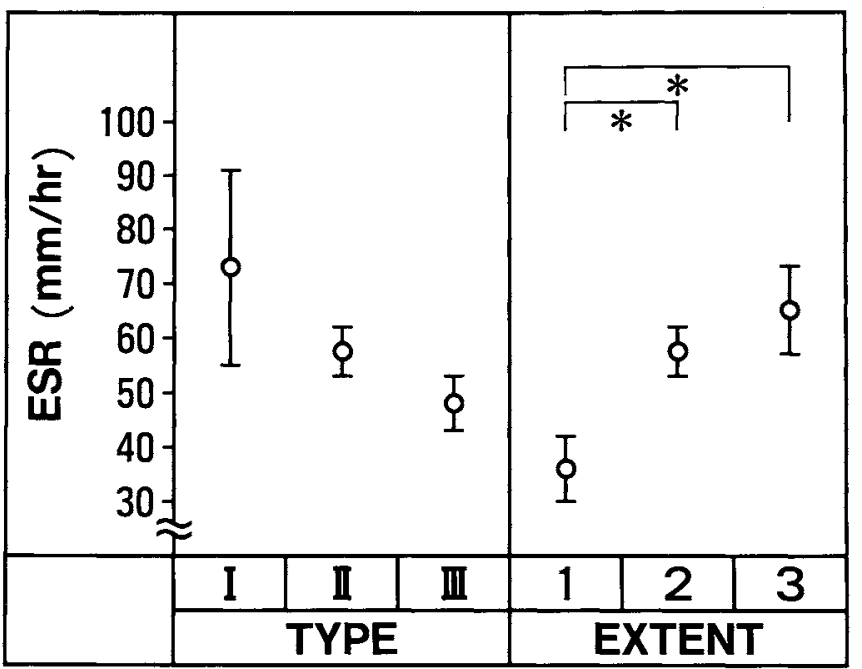

Fig. 6. Relationship between type, extent and erythrocyte sedimentation rate. circle, average; bar, standard error. ${ }^{*} \mathrm{p}<0.05$.

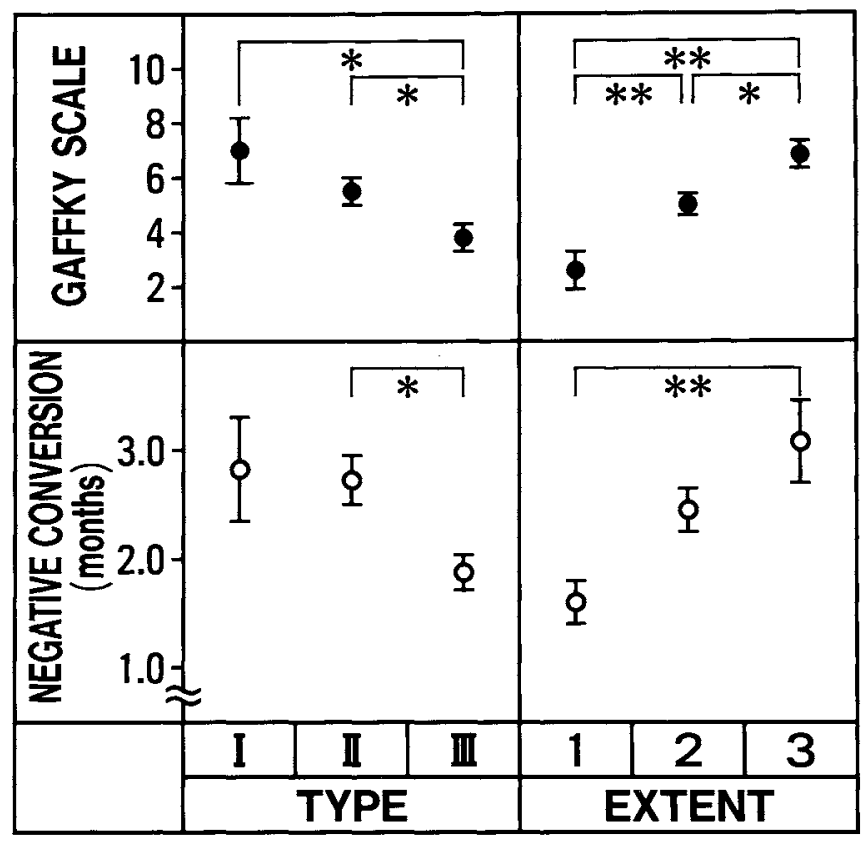

Fig. 7. Relationship between type, extent, Gaffky scale of sputum and negative conversion term of sputum culture. circle, averagc; bar, standard error. ${ }^{*} \mathrm{p}<0.05,{ }^{* *} \mathrm{p}<0.01$

does not decrease, the proliferative responses of $T$ and $B$ cells by specific antigens or non-specific mitogens are reduced in aged people $(3,6)$. This degenerative immunity likely affects the clinical features of tuberculosis such as cavity formation ( $3-5)$.

Malnutrition causes reduced cellular immunity apart from aging $(7,8)$. Tuberculin conversion by Bacille Celmette-Guérin vaccination is induced infrequently in malnourished people compared to well nourished (7, 9). Lymphopenia is often observed in the malnourished (8). Tuberculous patients frequently have emaciation, 


\section{Murate et al}

reduced serum albumin concentration and plasma amino acid imbalance (10-12). Lymphocyte transformation, delayed-type hypersensitivity, natural killer cell activity and interleukin-2 productivities are related to nutritional status in tuberculosis patients (12). In particular, the serum albumin concentration is closely related with type and extent.

Lymphopenia, neutrophil leukocytosis and monocytosis frequently occur in tuberculosis patients. They are closely associated with respiratory failure or tuberculosis death $(10,13)$. Lymphopenia is severe in the extended cases. Monocytes in tuberculosis patients suppress the blastogenic response to tuberculin purified protein derivative and produce a large amount of interleukin-1 (14). The development of tuberculous lesions results in an immunosuppressive state. Extended, malnourished, elderly, lymphopenia or pleurisy cases show reduced tuberculin reactions (15). Double-contour erythema by tuberculin skin test is negatively related to extent.

It is important for the treatment of tuberculosis patients to predict the amount of mycobacterial bacilli excreted and the time period for negative conversion. Both the Gaffky scale and negative conversion term markedly differ by type and extent. ESR well reflects the extent. Patients with no ESR elevation generally have a good course. Here we adopted many clinical parameters that are used in daily medical practice. They well reflect the differences in chest film appearances. Therefore, the "Gakkai Classification" is useful for the prediction of the risk of infection and the prognosis of active pulmonary tuberculosis.

Acknowledgments: We thank Professor Hidehiko Saito, First Department of Internal Medicine, Nagoya University School of Medicine, for his appropriate suggestions in the course of the present study.

\section{References}

1) The Committee for Classification of Tuberculosis in the Japanese Society for Tuberculosis (Nippon Kekkaku-byo Gakkai Byokei Bunrui Iinkai). The criteria for chest roentgenogram classification of pulmonary tuberculosis established by the Japanese Socicty for Tuberculosis (Nippon Kekkaku-byo Gakkai no Hai-kekkakushou X-sen Bunrui). Kekkaku 34: 885, 1959.

2) Edwards D, Kirkpatrick CH. The immunology of mycobacterial diseases. Am Rev Respir Dis 134: 1062, 1986.

3) Miyazaki N, Ogushi O, Higuchi K, Nakanishi $Y$, Kohashi $O$, Shigematsu N. Clinicopathological and immunological studies of pulmonary tuberculosis in the aged. Kekkaku 58: 427, 1983.

4) Harada S, Takamoto M, Harada $Y$, et al. Clinico-immunological studies of pulmonary tuberculosis in the elderly. Kekkaku 64: $529,1989$.

5) Shimokata $K$, Murate $T$, Ohgimi $T$, et al. Clinical characteristics of tuberculosis in the aged. Kakkaku 64: 649, 1989.

6) Negoro $S$, Hara $H$, Deguchi $Y$, et al. The characteristic changes of immune function with aging. Kekkaku 64: 657, 1989.

7) McMurray DN, Loomis SA, Casazza LJ, Rey H, Miranda R. Development of impaired cell-mediated immunity in mild and moderate malnutrition. Am J Clin Nutr 34: 68, 1981.

8) Bistrian BR, Blackburn GL, Scrimshaw NS, Flatt JP. Cellular immunity in semistarved states in hospitalized adults. Am J Clin Nutr 28: 1148, 1975.

9) . Chandra RK. Nutrition, immunity, and infection: present knowledge and future directions. Lancet 1: 688, 1983.

10) Onwubalili JK, Scott GM. Immune status in tuberculosis and response to treatment. Tubercle 69: 81, 1988

11) Maekawa J, Mikami R, Yoshikawa M, et al. Nutritional assessment of active pulmonary tuberculosis patients. Kekkaku 61: $379,1986$.

12) Yoneda T. Relation bctween malnutrition and cell-mediated immunity in pulmonary tuberculosis. Kekkaku 64: 633, 1989.

13) Barnes PF, Leedom JM, Chan LS, et al. Predictors of short-term prognosis in patients with pulmonary tuberculosis. J Infect Dis 158: $366,1988$.

14) Ellner JJ, Wallis RS. Immunologic aspects of mycobacterial infections. Rev Infect Dis 11: S455, 1989.

15) Kurasawa $T$. Tuberculin skin test of patients with active pulmonary tuberculosis and non-tuberculous pulmonary disease. Kekkaku 65: $47,1990$. 